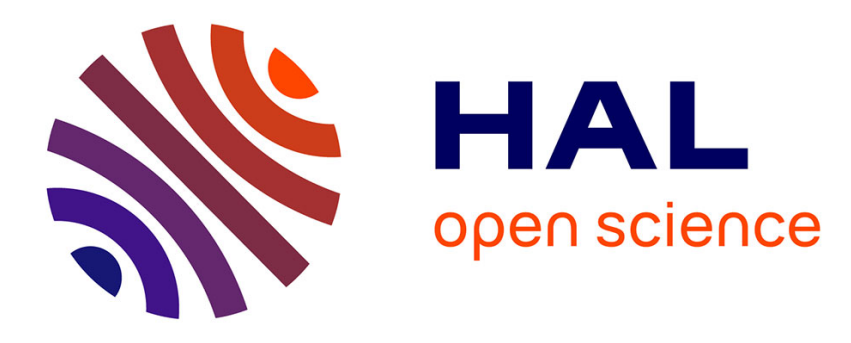

\title{
Les NTIC dans les PME: Stratégies, capacités organisationnelles et avantages concurrentiels
}

Serge Amabile, Martine Gadille

\section{To cite this version:}

Serge Amabile, Martine Gadille. Les NTIC dans les PME: Stratégies, capacités organisationnelles et avantages concurrentiels. Revue Française de Gestion, 2003, 3 (144), pp.43-63. 10.3166/rfg.144.4363. halshs-00348303

\section{HAL Id: halshs-00348303 \\ https://shs.hal.science/halshs-00348303}

Submitted on 18 Dec 2008

HAL is a multi-disciplinary open access archive for the deposit and dissemination of scientific research documents, whether they are published or not. The documents may come from teaching and research institutions in France or abroad, or from public or private research centers.
L'archive ouverte pluridisciplinaire HAL, est destinée au dépôt et à la diffusion de documents scientifiques de niveau recherche, publiés ou non, émanant des établissements d'enseignement et de recherche français ou étrangers, des laboratoires publics ou privés. 


\section{Les NTIC dans les PME : \\ Stratégies, capacités organisationnelles et avantages concurrentiels ${ }^{1}$}

\section{NICT in SMEs: \\ Strategies, Organizational Capabilities and competitive advantage}

\section{Résumé :}

Les résultats de recherche dont on rend compte ici s'intéressent aux conditions de création d'avantages concurrentiels en terme de coûts, de qualité et de nouveaux produits ou services à partir des usages de NTIC dans les PME. La capacité d'obtention d'une combinaison de ces trois sources d'avantages concurrentiels sur la base de modalités d'usage de NTIC, est expliquée par l'insertion de ces usages dans des contextes organisationnels, stratégiques et industriels spécifiques que l'on précise.

Serge AMABILE (Maître de Conférences, Sciences de Gestion) GRASCE (GREQAM UMR CNRS)

Faculté d'Economie Appliquée - Université d'Aix-Marseille III

15, 19 Allée C. Forbin

13627 Aix-en-Provence Cedex

Tél. : 04-42-96-14-96

Fax : 04-42-23-39-28

serge.amabile@,fea.u-3mrs.fr

Martine GADILLE (Chargée de recherche, Economie et Gestion)

LATAPSES-IDEFI (UMR CNRS-Université de Nice).

1 rue A. Einstein, Sophia Antipolis

06560 Valbonne

Tel : 0608840683

gadille@,univ-aix.fr

1 Les auteurs remercient vivement Alain d'Iribarne (Directeur de recherches au LEST - CNRS, Aix-enProvence -) pour l'encadrement scientifique du projet de recherche européen DEVNET qui a permis de réaliser ce papier. 


\section{INTRODUCTION}

Compte tenu du rôle joué par la petite entreprise dans une économie de la diversité (Piore et Sabel, 1984), les connaissances sur leurs modalités d'obtention d'avantages concurrentiels, à partir de l'usage de NTIC, constituent un enjeu du point de vue des Sciences de Gestion comme des Sciences Economiques (Foray et Mairesse, 1999). Cette question est d'autant plus importante que ces entreprises sont supposées ne pas posséder les ressources internes, notamment humaines, pour exploiter les possibilités de valorisation que les NTIC représentent (Brousseau et Rallet, 1999).

La présente recherche vise à préciser les processus et les contextes de construction d'avantages concurrentiels à la fois en terme de coûts, de qualité et d'innovation de produits ou services. Dans cette perspective, le dispositif d'enquête retenu cherche à cerner les relations entre stratégies des PME, contextes organisationnels et modes d'usages des NTIC $^{2}$ (Amabile, Gadille et Meissonier, 2000). Sur la base d'une population de départ (soit 5000 PME recensées à partir de fichiers des Chambres de Commerce et d'Industrie - CCI - des Pays de Loire), trois niveaux d'enquête successifs s'imbriquent. Ils articulent deux enquêtes statistiques et des études monographiques reposant sur des échantillons de tailles décroissantes correspondant à l'affinement de la population de départ. Les résultats d'étape présentés ici sont issus de la deuxième enquête statistique exploratoire réalisée par entretiens téléphoniques auprès des responsables de $108 \mathrm{PME}$.

Nous présentons d'abord la problématique de la recherche, le dispositif d'enquête, ainsi que les caractéristiques principales de l'échantillon de PME, au regard des différents types d'avantages concurrentiels obtenus au moyen des NTIC. On propose ensuite une analyse comparative entre d'une part, les PME qui déclarent obtenir par leurs usages des NTIC des résultats à la fois en termes de réduction des coûts, de qualité et d'innovation et d'autre part, les PME qui ne déclarent aucun résultat de ce type. Cette comparaison est conduite selon différentes dimensions : sectorielle, technologique, stratégique et organisationnelle. Ce travail permet d'élargir la définition d'avantage concurrentiel tel que cela a été suggéré par certains auteurs (Hamel et Prahalad, 1989; Bernasconi, 1996). En ce sens, nous intégrons plus particulièrement les capacités de l'organisation pour améliorer ses compétences et gérer des liaisons complexes dans une perspective d'innovation. Enfin, cet article permet de définir des pistes de recherche sur la viabilité des PME dans une économie de marchés concurrentiels et en constant renouvellement du point de vue des produits et des services.

\section{AdOPtion D'INTERNET, USAGES DES NTIC ET AVANTAGES CONCURRENTIELS}

Le dispositif d'enquête s'est appuyé sur un modèle d'analyse adapté aux PME. Nous nous sommes partiellement appuyés sur le modèle de production d'avantages concurrentiels de Porter ainsi que sur des conceptualisations de l'entreprise puisées dans les théories évolutionnistes ou des organisations. Sur cette base conceptuelle, l'objectif est de distinguer

\footnotetext{
2 Dans cet article, nous désignerons par Nouvelles Technologies de l'Information et de la Communication (NTIC) l'ensemble des technologies qui sont associées à l'usage d'Internet et de ses protocoles ainsi que les différentes formes de réseaux locaux reliant des micro-ordinateurs ou des stations de travail.
} 
principalement deux types de sous-populations : les PME qui n'obtiennent pas d'avantage concurrentiel à partir de leurs stratégies d'usages des NTIC et de leurs usages effectifs et celles qui déclarent en bénéficier, en cumulant simultanément des avantages en terme de coûts, de qualité et d'innovation de produits ou services.

\subsection{Modèle d'analyse et dispositif d'enquête}

L'usage d'Internet et des technologies de l'information et de la communication qui lui sont connexes (NTIC), peut favoriser, sous certaines conditions, l'obtention d'avantages concurrentiels du type réduction des coûts ou différenciation (Porter, 1985 ; Porter et Millar, 1985). Toutefois, ces avantages concurrentiels, s'ils peuvent résulter de stratégies génériques s'inscrivent surtout dans des aspects processuels d'organisation sur les bases de règles et pratiques héritées du passé. Ce point de vue est soutenu par de nombreux auteurs dont March (1991) qui mobilise pour cela les notions d'exploration et d'exploitation pour spécifier celle d'apprentissage organisationnel.

\subsubsection{Une analyse de la production d'avantages concurrentiels}

Selon Porter (1985), trois grandes stratégies génériques peuvent être envisagées pour créer et soutenir un avantage concurrentiel dans son secteur : la stratégie par les coûts, la stratégie par différenciation, la stratégie qui consiste à adopter l'une des deux premières, mais ciblée en fonction de segments spécifiques de l'activité. La mise en œuvre de chacune de ces différentes stratégies implique une trajectoire de recherche d'avantages concurrentiels spécifique.

Les notions de processus d'exploitation et d'exploration proposées par March (1991) en relation avec le concept d'apprentissage organisationnel, rejoignent par certains aspects celles développées par Porter tout en relativisant le déterminisme stratégique que l'on retrouve parfois dans les travaux de ce dernier. D'un côté, la notion d'exploitation désigne des processus de rationalisation et de production, de choix et de sélection, de mise en ouvre et d'exécution. D'un autre côté, la notion d'exploration est exprimée par des vocables du genre : recherche, variation, prise de risque, expérimentation, jeu, flexibilité, découverte, innovation.

En outre, March précise que les organisations font des choix implicites et explicites entre des processus d'exploitation et d'exploration. La compréhension de la sélection entre les deux processus et l'amélioration de leur équilibre est complexe. Les choix explicites sont basés sur des décisions calculées au sujet des investissements alternatifs et des stratégies en concurrence. Les choix implicites résultent des interactions entre de nombreux traits et coutumes organisationnels (par exemple entre les règles de recherche et les pratiques). Ils proviennent également de la façon dont les objectifs sont établis ou modifiés et dans les systèmes d'incitations.

Cependant, les points de vue de Porter et March convergent pour exprimer que la recherche d'avantage concurrentiel, selon des stratégies génériques pour l'un et des processus d'exploitation et d'exploration pour l'autre, sont en compétition dans l'allocation des ressources et la construction des compétences. De même, pour ces deux auteurs, le maintien d'un équilibre approprié entre les deux au niveau global de l'entreprise est un facteur primordial dans la survie et la prospérité des systèmes.

Les éléments théoriques précédents méritent à notre sens d'être complétés et adaptés à l'analyse de l'innovation dans les PME. En premier lieu, les stratégies de réduction des coûts 
et de différenciation ne paraissent pas suffisantes pour qualifier les différents modes de recherche d'avantage concurrentiel, appuyés sur les NTIC, de la PME. Porter et Millar suggéraient - dès 1985 - que, si les NTIC permettaient de prendre de l'avance sur la concurrence (du point de vue des coûts comme de la différenciation), elles permettaient également de modifier - par extension géographique ou industrielle - le champ de l'action concurrentielle, ce qui correspond, pour une large part, à des formes de diversification. D'autres auteurs (Kalika, 2000) préciseront ensuite que ces nouvelles technologies peuvent être tenues pour l'un des vecteurs de diversification vers de nouvelles activités. Ce point de vue est très bien synthétisé par Paturel (1997) selon lequel la diversification, en tant que manœuvre stratégique, correspondrait dans le cas le plus parfait, à l'entrée dans des activités se caractérisant par une technologie, des produits et des marchés nouveaux pour la firme. Il semble donc important d'introduire la notion de stratégie de diversification de l'activité qui n'a pas systématiquement le même sens en théorie et en pratique dans le cadre d'une PME que dans celui d'une grande entreprise ${ }^{3}$. Cette notion ne se confond pas avec celle de différenciation qui exprime surtout des transformations en termes de qualité des produits et de marketing, entendues dans un sens restreint par rapport à la création de nouveaux produits.

Dans le cas des PME, l'obtention d'avantages concurrentiels dans l'usage des NTIC peut donc s'exprimer en termes de réduction des coûts, de différenciation ou de diversification, en particulier des produits et services. De fait, il est potentiellement intéressant d'étudier les caractéristiques des entreprises parvenant à combiner effectivement, à partir de leurs usages des NTIC, ces trois types d'avantages concurrentiels. Notons que pour répondre à cette question on ne s'est pas centré sur les relations directes entre des stratégies génériques et les avantages concurrentiels de différents types. Partant de l'hypothèse que ces stratégies génériques sont médiatisées par des stratégie d'usages et des capacités organisationnelles nous nous sommes centrés sur les relations entre ces deux dernières dimensions et les types d'avantages concurrentiels obtenus.

Ce questionnement nous conduit à mobiliser de préférence un cadre théorique évolutionniste, plus large que celui qui se réfère à l'économie des coûts de transaction. La productivité et les coûts ne sont pas, en effet, les seuls motifs ni les seuls critères de production d'avantage concurrentiel à l'aune desquels les retours sur l'investissement dans l'usage d'Internet et des NTIC peuvent être mesurés. Les opportunités ou options d'achat, de développement et d'usages sont peut-être moins liées aux coûts des technologies qu'au manque d'apprentissage ou, simplement, de connaissance des usages possibles des NTIC. En ce sens, l'apprentissage par l'usage permet de faire évoluer deux choses : les cadres cognitifs de la prise de décision, donc les connaissances, à différents niveaux hiérarchiques, ainsi que les compétences mises en œuvre. Ainsi, les choix stratégiques restent médiatisés par des "capacités organisationnelles" (Chandler, 1992) ou "capacités dynamiques" (Nelson 1991; Teece et Pisano, 1998) que l'entreprise a pu construire à partir de sa trajectoire passée. Les avantages concurrentiels que peut obtenir une entreprise au moyen de nouvelles technologies sont donc liées, pour partie, au phénomène d'apprentissage par l'usage de ces technologies (Rosenberg, 1982) ${ }^{4}$ sur la base

\footnotetext{
${ }^{3}$ Dans le cas de la grande entreprise, la notion de diversification est, aujourd'hui, utilisée essentiellement pour désigner une opération financière d'achat de nouvelles activités portées par d'autres organisations. Elle renvoie seulement dans une moindre mesure à l'idée de création endogène de nouvelles activités à laquelle se réfèrent principalement les approches évolutionnistes de l'innovation. Dans le cas de la PME, la notion de diversification signifie principalement une transformation de l'activité endogène de l'entité organisationnelle et juridique de petite taille.

4 Précisons, qu'à travers l'expression "nouvelles technologies", Rosenberg ne désignait pas uniquement les NTIC même si celles-ci sont intégrées dans ses développements.
} 
de ses capacités organisationnelles. En s'appuyant sur ces éléments théoriques, on propose le modèle d'analyse suivant.

\subsubsection{Modèle d'analyse de la production d'avantage concurrentiel à partir des usages des NTIC}

Le schéma 1 met en relation cinq dimensions que nous proposons de mobiliser pour analyser les conditions d'obtention d'avantages concurrentiels par l'usage de NTIC. Le premier bloc représente l'hypothèse d'existence pour des PME des trois types d'actions stratégiques que nous avons déjà évoquées. Le deuxième bloc met en perspective que ces stratégies s'appuient, sous certains aspects volontairement, sur des infrastructures technologiques dont le système technique d'information et de communication constitue aujourd'hui un élément essentiel. Celui-ci est utilisé en fonction de stratégies d'usages ${ }^{5}$ pouvant être initiées par le management et/ou les utilisateurs (troisième bloc). Ces usages peuvent se réaliser avec des partenaires à l'intérieur ou à l'extérieur de l'entité organisationnelle considérée, selon des applications et des domaines d'activité divers (ce que nous préciserons par la suite). La relation entre les stratégies génériques et les stratégies d'utilisation du système technique d'information et de communication est elle-même dépendante des capacités organisationnelles et collectives d'appropriation de ces technologies. Inversement, ces dernières sont aussi dans le temps transformées par les interactions entre ces différentes stratégies, dont une part relève de résultats non intentionnels.

Comme nous l'avons déjà observé, les capacités organisationnelles sont définies par des combinaisons entre des processus d'exploration et d'exploitation de routines. S'agissant des usages des NTIC qui nous concernent directement, ces processus entretiennent une relation d'interdépendance dynamique dans le temps : la recherche de nouvelles règles d'usage se fait sur les bases d'anciennes et se traduit dans le temps par de nouvelles routines. Ces interactions se concrétisent par l'évolution des compétences et des connaissances en matière de nouvelles possibilités d'usages ou de nouvelles possibilités technologiques. Cette évolution peut rétroagir sur le processus de prise de décision stratégique à partir des compétences, des connaissances et plus largement des "cadres interprétatifs" du management.

De l'ensemble de ces interdépendances temporelles (Gaffard, 1990) qui médiatisent les stratégies génériques initiales, découlent des effets de compétitivité que nous proposons d'évaluer en terme de réduction des coûts, de différenciation (par la qualité) et de diversification (création de nouveaux produits/services).

\footnotetext{
5 Nous verrons dans la deuxième partie de cet article que ces stratégies d'usages peuvent correspondre à la mise en place de pratiques et d'applications de veille économique, de marketing, etc.
} 


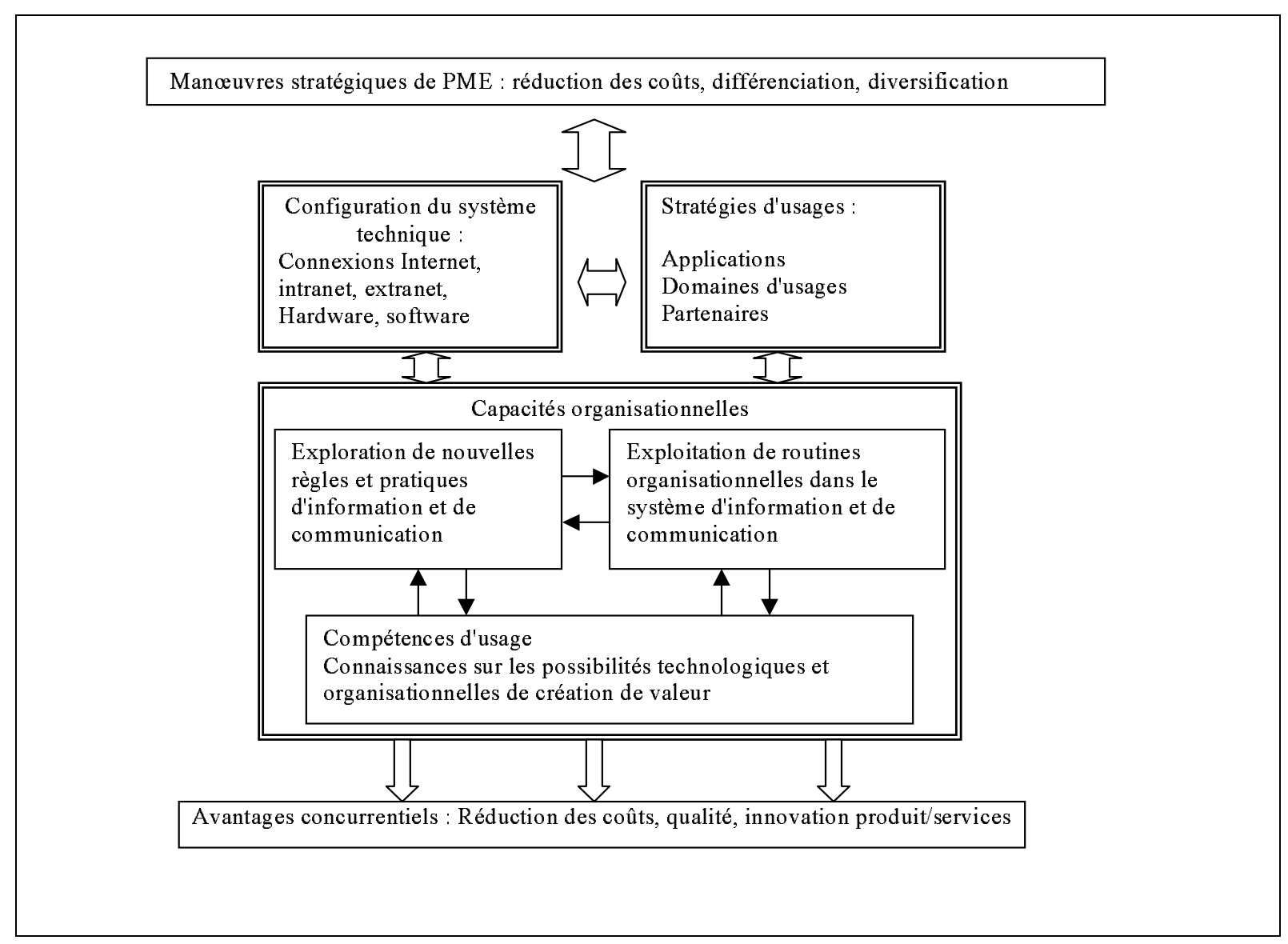

Figure 1 : NTIC, apprentissages par l'usage et recherche d'avantage concurrentiel

\subsection{Dimensions et indicateurs du questionnaire}

La construction du questionnaire reprend les dimensions principales évoquées ci-dessus et permet de tester leurs interrelations dans l'obtention d'avantages concurrentiels analysées selon les trois dimensions: productivité et réduction des coûts, différenciation en qualité, diversification des produits et services.

\subsubsection{La configuration du système technique et son évolution}

Une première dimension permet de caractériser le niveau et la stratégie de configuration des équipements informatiques: nombre d'ordinateurs, types de réseaux, fonctions et acteurs de l'entreprise équipés pour utiliser les accès réseaux.

Elle prend également en compte les projets d'évolution du système technique, son évaluation par le responsable de l'entreprise ainsi que le moment de l'adoption d'Internet. Sont donc demandées dans cette perspective les dates de découverte des usages possibles de l'Internet et de la première connexion.

\subsubsection{Les stratégies d'usages}

Les stratégies d'usage sont d'abord repérées à partir d'une question sur le ou les motifs de la décision de la connexion à Internet. Afin de mieux lier ces stratégies aux capacités organisationnelles de la PME, il a également été demandé si le ou les acteurs à l'origine de l'utilisation de l'Internet et de la mise en réseau des ordinateurs étaient des acteurs internes, 
externes ou les deux. Cette dernière question donnait un point de vue supplémentaire sur les capacités internes mobilisables et/ou mobilisées dans l'organisation.

Les stratégies d'usages sont aussi analysées à partir de l'existence déclarée d'applications utilisées ou développées par l'entreprise autour d'Internet et des NTIC : le mail, l'échange de fichier, la consultation et/ou le développement de sites $W e b$, les applications de commerce électronique, de marketing, de veille, etc. L'évolution des projets de développement et d'usage de NTIC a également été abordée afin de mesurer la cohérence entre les capacités organisationnelles et les projets.

La mobilisation des NTIC pour médiatiser les relations de l'organisation avec différents types de "partenaires externes" peut être à la fois perçue comme un révélateur des stratégies d'usages et des possibilités de capter des externalités de réseaux agissant sur les capacités organisationnelles.

La mise en relation des stratégies d'usages avec le système technique permet de saisir la cohérence stratégique entre la configuration du système technique et celle des usages "internes" comme "externes". Ces deux premières dimensions sont regardées dans leur relation d'interdépendance avec les capacités organisationnelles et leur évolution.

\subsubsection{Les capacités organisationnelles et leur évolution}

Les capacités organisationnelles sont repérées à partir de plusieurs sous-dimensions :

- les changements organisationnels (formels/informels) qui sont intervenus ou non pour favoriser l'usage des NTIC. L'occurrence de ces changements renseigne sur la nature de l'équilibre entre processus d'exploitation et d'exploration ;

- l'accès à des informations sur l'entreprise ou son environnement par les salariés et la transmission de l'information obtenue au moyen de NTIC entre les services ou les employés ;

- l'apport des technologies de l'information dans les processus de communication, de décision et d'organisation ;

- la construction des compétences d'usage et de maintenance : l'entreprise s'est-elle donné les moyens de ces processus d'exploration et d'exploitation, quelles sont les procédures à l'œuvre concernant la formation des ressources humaines?

- la représentation par le responsable de PME du potentiel de compétences qu'il détient pour les usages en cours et les usages futurs.

\subsubsection{La contribution d'Internet et des NTIC à la compétitivité de l'entreprise}

Sont considérés comme des facteurs de second rang pouvant agir sur la compétitivité de l'entreprise : la réactivité aux marchés, l'élargissement du portefeuille clientèle, l'augmentation du nombre de partenaires externes avec lesquels l'entreprise est reliée via les NTIC. Le questionnaire comprend donc un jeu d'items de cette nature. 
Ensuite, afin de recueillir l'opinion ${ }^{6}$ des responsables d'entreprises sur les trois types d'avantages concurrentiels, des questions directes sont posées quant à :

- l'effet des NTIC sur la productivité et la réduction des coûts ;

- la différenciation en termes d'amélioration de la qualité ;

- la diversification des produits et services.

\subsection{La mise en œuvre de l'enquête et constitution de sous-populations}

\subsubsection{Le dispositif}

Nous avons déjà précisé que les résultats présentés dans cet article proviennent du deuxième niveau d'enquête statistique réalisée, sous la forme d'une enquête téléphonique, au cours du premier semestre de l'année 1999, dans le cadre du projet européen DEVNET $^{7}$. Les 108 entreprises ainsi étudiées font partie d'un premier échantillon de $600 \mathrm{PME}$, toutes connectées à Internet, et ayant initialement répondu à une enquête postale réalisée au cours du second semestre de 1998. Cet échantillon avait été constitué principalement en fonction de 2 critères : la taille (les firmes ayant un nombre de salariés inférieur à 400 ont été retenues ${ }^{8}$ ) et la localisation géographique (ont été retenues, des entreprises des régions de la Loire et du Poitou-Charentes) en relation avec les hypothèses d'effet territorial sur la capacité des PME à s'approprier des usages de NTIC. Ce dispositif permet d'approfondir les champs d'interrogations mis en perspective à la suite du questionnaire postal (Gadille, Iribarne 2000).

\subsubsection{Critères de constitution des sous-populations}

Selon les travaux précédemment mobilisés dans cet article, nous retiendrons que l'utilisation des NTIC soutient, sous certaines conditions, la réalisation de gains de productivité, la réduction des coûts et des délais de production et de transaction, par exemple en matière de télécommunication. Elle peut également favoriser une adaptation de la qualité des produits ou services existants. Enfin, elle peut favoriser la diversification en terme de produits et services. Cette diversification peut permettre à l'entreprise de rester unique sur le marché, elle repose sur une capacité de compréhension, d'adaptation, voire de création de nouveaux marchés ${ }^{9}$. Les NTIC sont alors, potentiellement, mobilisables comme instruments de veille économique, marketing ou technologique et législative. En ce sens, elles développent l'accessibilité d'informations auxquelles l'entreprise pouvait difficilement parvenir auparavant ou, plus simplement, elles peuvent améliorer l'accès à certaines informations. L'utilisation d'un site, l'usage d'applications de marketing sur Internet, une activité de veille économique et

\footnotetext{
6 A chaque fois que cela c'est révélé possible et pertinent, nous avons cherché à approfondir les réponses recueillies au sein des entreprises enquêtées. En effet, prétendre utiliser ou, a fortiori, valoriser les NTIC - en particulier Internet - peut conférer une "étiquette" d'entreprise innovante parfois recherchée par les dirigeants de PME. Ainsi, en "surfant" sur le Web, nous avons pu vérifier l'existence d'un site pour les responsables déclarant en avoir fait développer un dans leur organisation, envoyer des messages aux différentes adresses électroniques signalées par les répondants, etc.

7 Ce projet a été financé par le Fond Social Européen et piloté par la Chambre Régionale de Commerce et d'Industrie (C.R.C.I.) des pays de la Loire.

8 On remarque que les très petites PME n'ont pas été exclues de l'échantillon : 27 entreprises dont le nombre de salariés est inférieur à 11 ont été conservées.

${ }^{9}$ Le projet de cet article n'étant pas de développer une présentation des stratégies de diversification, nous renvoyons le lecteur vers des ouvrages spécialisés comme, par exemple, celui de P. Very, Stratégies de diversification, Editions Liaisons, 1991.
} 
technologique peuvent être des supports importants de la stratégie de diversification de la PME. Toutefois, ils peuvent également relever des deux stratégies précédentes. En ce sens, il semble intéressant d'appréhender et de relier les facteurs et les contextes organisationnels associés à ces différentes stratégies avec les usages des NTIC.

De fait, quatre grands types de PME, sont identifiés dans un premier temps, en fonction de leurs capacités à obtenir des avantages concurrentiels selon les deux critères que sont la réduction des coûts et la diversification. Un premier filtre est ainsi opéré avec les indicateurs d'opinion, d'un côté sur les gains de productivité et la réduction des coûts et d'un autre côté sur la diversification. Pour faciliter l'analyse, la différenciation par la qualité est ensuite utilisée comme indicateur de second rang dans la réalisation d'avantages concurrentiels.

En termes de résultats et selon la combinatoire des deux types d'avantages concurrentiels retenus en premier rang, $43 \%$ des $\mathrm{PME}$ (44 en $\mathrm{VA}^{10}$ ) déclarent avoir effectué ni diversification, ni gains de productivité ou réduction des coûts, à partir de leur usage de NTIC. Ensuite 32\% déclarent effectuer seulement des gains de productivité ou des réductions de coûts tandis que $11 \%$ déclarent effectuer seulement de la diversification de leurs produits ou services. Enfin, 14\% (15 en VA) des PME déclarent simultanément à partir de leurs usages des NTIC, obtenir une réduction de coûts et une diversification de produits et services.

La combinaison des deux types d'avantages concurrentiels est donc bien réalisée par certaines entreprises même si cela ne concerne qu'une minorité de PME enquêtées. Ce constat semble rejoindre les hypothèses de March et Porter sur la difficulté de combiner différents types d'avantage concurrentiel ${ }^{11}$.

Si l'on réintègre la création d'avantages concurrentiels à partir de la différenciation, les résultats déclarés en terme d'amélioration de la qualité (tableau 1), montrent que la population 1 , bénéficiant de réduction des coûts et de diversification des produits et services, cumule également une capacité plus importante quant à l'amélioration de la qualité. Ce résultat vient corroborer l'hypothèse selon laquelle les différents types d'avantages concurrentiels peuvent être combinés au sein d'une même organisation sous différentes conditions qui semblent interdépendantes et qui restent à préciser.

Utilisation des NTIC et amélioration

de la qualité des services ou de produits

\begin{tabular}{|r|c|c|c|c|}
\cline { 2 - 5 } \multicolumn{1}{c|}{} & \multicolumn{2}{c|}{ Population 1 } & \multicolumn{2}{c|}{ Population 2 } \\
\hline Pas d'accord, plutôt pas d'accord & 1 & $7 \%$ & 25 & $56 \%$ \\
\hline D'accord, plutôt d'accord & 13 & $87 \%$ & 17 & $38 \%$ \\
\hline Ne sait pas & 1 & $6 \%$ & 3 & $6 \%$ \\
\hline
\end{tabular}

\section{Strategies d'usage des NTIC, Capacites organisationnelles et OBTENTION D'AVANTAGES CONCURRENTIELS}

Cette partie apporte des éléments sur la nature industrielle et les caractéristiques stratégiques et organisationnelles des deux sous-populations d'entreprises : celles qui réalisent principalement les deux types d'avantages concurrentiels - réduction des coûts et diversification des produits et services, population 1, 15 répondants - et celles qui déclarent ne réaliser aucun de ces deux types d'avantages concurrentiels - population 2, 44

\footnotetext{
10 Précisons que les pourcentages sont sensiblement équivalents aux valeurs absolues (VA), en fonction des non-réponses, puisque le nombre d'entreprises interrogées était de 108.

11 Rappelons toutefois que pour le deuxième type d'avantage concurrentiel, M. Porter évoquait la différenciation et non la diversification comme nous le proposons.
} 
répondants -. Compte tenu de la faiblesse des effectifs dans la première population, il s'agit bien d'une phase exploratoire en terme d'hypothèses et de problématique ${ }^{12}$.

La comparaison entre ces deux sous-populations permet, in fine, de repérer les effets de dépendance du chemin et d'apprentissages par l'usage tels qu'ils sont supposés par Rosenberg (1982).

\subsection{Nature sectorielle et création d'avantages concurrentiels}

On précise la nature industrielle des PME dans les deux sous-populations, afin didentifier au préalable l'existence de facteurs discriminants en faveur de l'obtention ou non d'avantages concurrentiels sur la base des usages des NTIC. Pour cela, on propose de comparer les tailles, les secteurs, les taux d'équipements, les dates de connexion à Internet ainsi que les caractéristiques des réseaux informatiques et télécommutés.

Le tableau sur les effectifs de ces PME permet de suggérer (vue la stabilité entre les 2 populations) que le nombre d'employés ne pourra - dans nos interprétations ultérieures constituer une base solide d'explication des différences de valorisation des NTIC entre les 2 sous-populations. Concernant la population 1, on note, en effet, que la capacité à réaliser les deux types d'avantages concurrentiels n'est apparemment pas liée à la taille de l'entreprise.

\section{Tableau 2 : Détails des effectifs ${ }^{13}$}

\begin{tabular}{|c|c|c|c|c|}
\cline { 2 - 5 } \multicolumn{1}{c|}{} & \multicolumn{2}{c|}{ Population 1 } & \multicolumn{2}{c|}{ Population 2 } \\
\hline 0 à 10 & 4 & $27 \%$ & 14 & $32 \%$ \\
\hline 11 à 30 & 3 & $20 \%$ & 8 & $18 \%$ \\
\hline 31 à 50 & 4 & $27 \%$ & 9 & $20 \%$ \\
\hline 51 à 401 & 4 & $27 \%$ & 13 & $30 \%$ \\
\hline
\end{tabular}

Par contre on observe une différence dans la nature des secteurs représentés entre les deux sous populations. En effet, le tableau 3 montre que les entreprises parvenant à allier réductions de coûts et diversification sont plus nombreuses dans le secteur des services au sein duquel le secteur des services aux entreprises joue un rôle moteur dans la dynamique d'adoption d'Internet (Gadille, Iribarne, 2000). En sens inverse, la population 2 se distingue par une sur représentation des secteurs les moins touchés (au moment de l'étude) par l'usage des NTIC : agriculture, construction, transport. On a donc affaire ici à un effet structurel de l'industrie sur la capacité de la PME à utiliser et valoriser les NTIC. Ces résultats rejoignent d'autres développements, obtenus sur la population mère, mettant en perspective que le secteur des services aux entreprises et des biens d'équipement tirent la dynamique de diffusion des NTIC (Gadille, Iribarne, 2000).

\footnotetext{
12 Nous avons délibérément choisi dans cet article de ne présenter que les tendances les plus significatives repérées au sein de ces 2 populations sans produire différents tableaux (de contingence, par exemple). Nous le verrons en conclusion, certains faits semblent suffisamment significatifs sans mobiliser des analyses multivariées

13 Dans ce tableau ainsi que dans les suivants, les pourcentages ont été arrondis afin d'augmenter la lisibilité des résultats présentés. Par ailleurs, pour chaque variable étudiée, les tableaux reprendront l'architecture suivante : la 1e colonne présente les différents items ou classes de la variable concernée (exemple: pour le

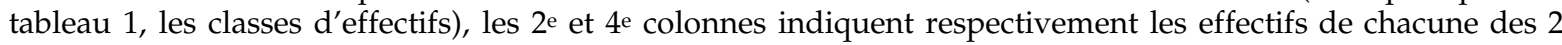
sous-populations en fonction des réponses obtenues alors que les $3^{\mathrm{e}}$ et $5^{\mathrm{e}}$ colonnes présentent les pourcentages correspondant à ces effectifs (respectivement pour la population 1 et la population 2).
} 


\begin{tabular}{|r|c|c|c|c|}
\cline { 2 - 5 } \multicolumn{1}{c|}{} & Population 1 & Population 2 \\
\hline Agriculture, transports, construction & 1 & $7 \%$ & 8 & $18 \%$ \\
\hline Industries & 2 & $13 \%$ & 14 & $31 \%$ \\
\hline Commerce & 2 & $13 \%$ & 6 & $13 \%$ \\
\hline Services & 10 & $67 \%$ & 17 & $38 \%$ \\
\hline Total répondants & 15 & & 45 & \\
\hline
\end{tabular}

\begin{tabular}{|r|c|c|c|c|}
\hline \multicolumn{1}{c}{} & Population 1 & Population 2 \\
\hline Accès Internet par ordinateur & 15 & $100 \%$ & 39 & $100 \%$ \\
\hline Réseau Intranet & 10 & $67 \%$ & 6 & $15 \%$ \\
\hline Réseau Extranet & 1 & $7 \%$ & 0 & $0 \%$ \\
\hline Réseau local ou propriétaire & 14 & $93 \%$ & 31 & $80 \%$ \\
\hline Total répondants & 15 & & 39 & \\
\hline
\end{tabular}

Les dates de connexion à Internet, qui renvoient en théorie à la notion d'adoption de la technologie, constituent un indicateur du temps passé pour l'apprentissage par l'usage et les possibilités de développement de nouveaux usages. Leur analyse révèle qu'il y a, de ce point de vue, peu de différence entre les deux populations et que si différences il y a, elles montrent que les entreprises ne créant pas d'avantages concurrentiels à partir de leurs usages des NTIC se sont équipées plus tôt ${ }^{14}$. De fait, le temps écoulé après l'adoption de la technologie ne peut être considéré comme la seule base de l'apprentissage et de la valorisation liés aux NTIC. Ces derniers semblent également provenir des modalités d'usages de et dans l'organisation.

Les équipements informatiques, mesurés dans un premier temps par le nombre d'ordinateurs moyen, ne constituent pas non plus une variable réellement discriminante pour l'obtention d'avantages concurrentiels entre les deux populations. Alors que la répartition en fonction de la taille des entreprises est homogène entre les populations 1 et 2 , ces dernières présentent des moyennes respectives de 28 et de 20 ordinateurs par PME.

En revanche, la présence d'Intranets semble différencier ces deux populations (sous réserve de la significativité du nombre d'entreprises concernées). En effet, un déséquilibre important apparaît sur le nombre d'Intranet développé ( 2 entreprises sur 3 dans la population 1, moins d'une entreprise sur 6 dans la population 2). Les entreprises de la population 1 s'approprieraient donc mieux la technologie intranet, connexe à Internet, qui permet d'échanger et de mutualiser des informations sur un réseau interne à l'organisation, ce qui apparaît cohérent avec une activité de service prédominante.

Il semble donc que la capacité de création d'avantages concurrentiels en termes de coûts et d'innovation de produits et services soit moins liée au fait de se connecter à l'Internet qu'au contexte technologique, sectoriel et organisationnel dans lequel l'entreprise se connecte. La dépendance du chemin évoquée par les évolutionnistes serait donc d'abord lié à la position du secteur d'appartenance de la PME par rapport à la diffusion du paradigme technologique. Néanmoins, cette dynamique sectorielle n'est pas suffisante pour expliquer la présence de PME appartenant à d'autres secteurs. Probablement, ce résultat suggère que les entreprises compétitives au moyen des NTIC savent généralement mieux articuler une technologie et une organisation tournée sur les échanges externes et une technologie et une organisation tournée sur les échanges internes. Il convient donc d'aborder les dimensions de stratégies d'usage et de capacités organisationnelles.

\subsection{Stratégies d'usages des NTIC et source d'avantages concurrentiels}

Les stratégies d'usage qui concernent tant le management que les utilisateurs en général, ne sont pas forcément homogènes et cohérentes dans l'entreprise, elles se distribuent selon des constructions spécifiques d'acteurs de l'organisation. Il s'agit ici d'évaluer le rôle de l'intentionnalité du dirigeant à partir des motifs d'adoption, ainsi que l'effet d'une volonté

14 13\% des entreprises de la population 1 ( 2 en VA) se sont connectées avant 1997 contre 19\% pour la population 2 (8 en VA) ; 80\% (12 en VA) de la population 1 se sont connectées entre janvier 97 et janvier 99 contre 74\% (32) pour la population 2 . Les $7 \%$ restant pour les deux populations se sont connectés après janvier 99 , année de l'enquête. 
stratégique en matière de développement d'applications liées aux NTIC. Pour approfondir ceci, nous abordons également l'usage dans l'organisation selon différentes activités et selon différents partenaires, et enfin l'aspect diffus ou non dans l'entreprise des accès réseaux.

\subsubsection{L'importance de l'intentionnalité du dirigeant dans les stratégies d'usage}

Pour évaluer dans quelle mesure les organisations concernées ont produit une réflexion sur elles-mêmes et quelle fut leur intentionnalité, nous avons posé la question en priorité aux managers ou à leurs proches collaborateurs concernant le ou les motifs à l'origine de la décision de la connexion à Internet.

Tableau 5 :

Motifs de la décision de la connexion à Internet

\begin{tabular}{|l|c|c|c|c|}
\cline { 2 - 5 } \multicolumn{1}{c|}{} & Population 1 & Population 2 \\
\hline pour voir & 3 & $20 \%$ & 23 & $55 \%$ \\
\hline par contrainte & 3 & $20 \%$ & 5 & $12 \%$ \\
\hline $\begin{array}{l}\text { dans le cadre d'un projet en vue d'applications } \\
\text { précises }\end{array}$ & 9 & $60 \%$ & 12 & $29 \%$ \\
\hline autre & 3 & $20 \%$ & 14 & $33 \%$ \\
\hline
\end{tabular}

Même si les populations étudiées ne sont pas statistiquement significatives, il paraît pertinent de souligner une double tendance : $60 \%$ des entreprises de la population 1 se sont connectés dans le cadre d'un projet en vue d'applications précises (par exemple, la mise en œuvre d'Echanges de Données Informatisés) alors que... 55\% des entreprises de la population 2 (soit 23 firmes) s'étaient connectées à Internet "pour voir" !

Le fait que les entreprises déclarant valoriser les NTIC soient majoritairement celles pour lesquelles un projet précédait la mise en place de ces dernières (la connexion à Internet tout au moins) mérite un approfondissement. C'est ce que nous proposons de faire en comparant les deux populations dans leurs stratégies de développement d'application.

Tableau 6 : Développement d'applications de "mise en relation et connaissance de l'environnement"

\begin{tabular}{|c|c|c|c|c|}
\hline & \multicolumn{2}{|c|}{ Population 1} & \multicolumn{2}{|c|}{ Population 2} \\
\hline A développé son site Web : Oui & 15 & $100 \%$ & 11 & $25 \%$ \\
\hline Non & 0 & $0 \%$ & 33 & $75 \%$ \\
\hline A développé des applications pour le marketing : Oui & 11 & $79 \%$ & 4 & $9 \%$ \\
\hline Non & 3 & $21 \%$ & 38 & $91 \%$ \\
\hline A développé des applications pour la veille économique : Oui & 6 & $55 \%$ & 5 & $12 \%$ \\
\hline Non & 5 & $45 \%$ & 37 & $88 \%$ \\
\hline
\end{tabular}

Les pourcentages semblent édifiants. Ils le sont d'avantage lorsqu'on vérifie que $87 \%$ des entreprises de la population 1 ont développé 2 des 3 applications citées... contre $16 \%$ pour les entreprises de la population 2. On note que les applications les plus discriminantes en faveur de la création d'avantages concurrentiels sont celles liées au marketing $(91 \%$ de non dans la population 2). Cette observation confirme l'hypothèse générale de mobilisation du système d'information pour capter les aspirations des clients et ainsi anticiper au mieux les évolutions de marchés (Benghozi, 1996 ; Bernasconi 1996).

\subsection{2. domaines d'usages, partenariat et externalités de réseaux}

Cette dynamique d'usage pour soutenir l'attention des PME à leurs environnements se retrouve également dans les domaines d'usages mobilisés dans leurs relations (médiatisées par les NTIC) avec un large spectre de partenaires. Le tableau suivant montre que, s'il y a une certaine stabilité sur la plupart des items, un écart manifeste apparaît quant à la rubrique 
prospection de nouveaux marchés et marketing. Cette différence d'intensité d'usage en faveur de ce dernier domaine semble aller de pair avec l'existence de stratégies de développement d'application marketing constatée au sein de la population 1 qui semble créer des avantages concurrentiels sur cette base. Cette tendance se retrouve au niveau de l'élargissement du portefeuille clientèle (tableau 8) : la population 1 qui réalise des réductions de coûts et une diversification de ses produits et services accroît également sa clientèle.

Tableau 7 : domaines d'utilisation des NTIC avec les partenaires (possibilité de plusieurs réponses)

\begin{tabular}{|c|c|c|c|c|}
\hline \multirow[b]{2}{*}{ Formation et formation à distance } & \multicolumn{2}{|c|}{ Population 1} & \multicolumn{2}{|c|}{ Population 2} \\
\hline & 1 & $7 \%$ & 1 & $2 \%$ \\
\hline R et D pour de nouveaux produits, services ou technologies & 7 & $47 \%$ & 9 & $21 \%$ \\
\hline $\begin{array}{l}\text { Suivi clientèle, assistance après vente, échange de documents avec le réseau } \\
\text { vente }\end{array}$ & 11 & $73 \%$ & 24 & $56 \%$ \\
\hline Achats/Ventes & 6 & $40 \%$ & 15 & $35 \%$ \\
\hline Gestion de production & 2 & $13 \%$ & 6 & $14 \%$ \\
\hline Comptabilité & 5 & $33 \%$ & 7 & $16 \%$ \\
\hline Prospection de nouveaux marchés, marketing & 12 & $80 \%$ & 8 & $19 \%$ \\
\hline Autres & 6 & $40 \%$ & 16 & $37 \%$ \\
\hline Total répondants & 15 & & 43 & \\
\hline
\end{tabular}

\begin{tabular}{|c|c|c|c|c|c|}
\hline \multirow{4}{*}{$\begin{array}{l}\text { Tableau } 8: \text { ' 'utilisation des NTIC a permis } \\
\text { à votre entreprise d'élargir votre portefeuille } \\
\text { clientèle }\end{array}$} & & \multicolumn{2}{|c|}{ Population 1} & \multicolumn{2}{|c|}{ Population 2} \\
\hline & Pas d'accord, plutôt pas d'accord & 6 & $43 \%$ & 35 & $80 \%$ \\
\hline & D'accord, plutôt d'accord & 8 & $57 \%$ & 4 & $9 \%$ \\
\hline & Ne sait pas & 0 & & 5 & $11 \%$ \\
\hline
\end{tabular}

Par ailleurs, si les entreprises des deux populations sont largement amenées à utiliser les NTIC avec différents types de partenaires (ceci dans des proportions relatives stables d'une population à l'autre), nous notons que $40 \%$ des entreprises de la population 1 (pour $12 \%$ des entreprises de la population 2) utilisent les NTIC pour échanger avec des organismes professionnels ou des organismes consulaires.

Tableau 9. Les acteurs externes avec lesquels les PME utilisent les NTIC (possibilité de plusieurs réponses)

\begin{tabular}{|l|c|c|c|c|}
\hline Clients & 13 & $87 \%$ & 33 & $82 \%$ \\
\hline Entreprises donneurs d'ordre & 6 & $40 \%$ & 10 & $25 \%$ \\
\hline Entreprises sous-traitantes & 5 & $33 \%$ & 5 & $12 \%$ \\
\hline Entreprises partenaires & 13 & $87 \%$ & 13 & $32 \%$ \\
\hline Etablissements, agence ou siège & 6 & $40 \%$ & 11 & $27 \%$ \\
\hline Laboratoires de recherche, organismes de formation supérieure & 3 & $20 \%$ & 1 & $2 \%$ \\
\hline Organismes professionnels ou organismes consulaires & 6 & $40 \%$ & 5 & $12 \%$ \\
\hline Collectivités territoriales, services déconcentrés de l'Etat & 2 & $13 \%$ & 3 & $7 \%$ \\
\hline Banques & 2 & $13 \%$ & 5 & $12 \%$ \\
\hline Autres dont fournisseurs & 2 & $13 \%$ & 4 & $10 \%$ \\
\hline Total répondants & 15 & & 40 & \\
\hline
\end{tabular}

$\mathrm{Ne}$ révèle-t-on pas ici un comportement de renseignement, d'écoute et d'ouverture sur l'environnement à travers des contacts avec les organismes consulaires et les organismes de formation supérieure (afin de trouver de nouvelles compétences, par exemple) et d'autres entreprises partenaires.

Il est possible d'apporter des éléments de réponse à cette question en observant que, tout en étant relativement plus en relation via les NTIC avec d'autres entreprises partenaires, les entreprises de la population 1 ont également trouvé de nouveaux partenaires grâce à l'usage des NTIC (57\% des entreprises de la population 1 contre $23 \%$ dans la population 2 ). 
Tableau 10 : L'utilisation des NTIC a permis à votre entreprise de...

\begin{tabular}{|r|c|c|c|c|}
\cline { 2 - 5 } ...trouver de nouveaux fournisseurs ou partenaires & \multicolumn{2}{|c|}{ Population 1 } & \multicolumn{2}{c|}{ Population 2} \\
\hline Pas d'accord, plutôt pas d'accord & 5 & $36 \%$ & 30 & $68 \%$ \\
\hline D'accord, plutôt d'accord & 8 & $57 \%$ & 10 & $23 \%$ \\
\hline Ne sait pas & 1 & $7 \%$ & 4 & $9 \%$ \\
\hline
\end{tabular}

Peut-on supposer qu'au sein de ces partenaires se retrouvent surtout des firmes avec lesquelles les organisations interrogées ont développé des processus de coopération (pour de la $\& \& D$, de la formation, etc.), des procédures de co-développement de produits, etc. On retrouverait là, en partie, l'aspect de construction sectorielle des entreprises de services aux entreprises dont l'utilisation d'Internet et la production de services liés aux NTIC est centrale dans leur production de valeur. Néanmoins cette appartenance sectorielle n'est pas une condition suffisante ni même nécessaire pour créer à partir de modalités d'usage des NTIC des avantages concurrentiels.

\subsection{Capacité organisationnelle et usages efficients des NTIC}

On suppose plus précisément que les options collectives de transformation de l'organisation, des relations de travail et des ressources par rapport à une trajectoire passée ne sont pas neutres dans la capacité d'obtention d'avantages concurrentiels sur la base d'usage d'Internet.

De façon limpide, les entreprises déclarant valoriser les NTIC (autant sur la diversification que sur la réduction des coûts de revient) sont majoritairement celles ayant procédé à des adaptations de leur organisation. On peut donc faire l'hypothèse que les firmes déclarant valoriser ces technologies ont remis en cause avant, pendant ou après l'adoption des NTIC, leur fonctionnement global d'une part, leur organisation du travail d'autre part.

Tableau 11 : Modification de l'organisation formelle de l'entreprise pour communiquer des informations obtenues via les NTIC

\begin{tabular}{|l|c|c|c|c|}
\cline { 2 - 5 } \multicolumn{1}{c|}{} & Population 1 & \multicolumn{2}{c|}{ Population 2} \\
\hline Pas d'accord, plutôt pas d'accord & 3 & $23 \%$ & 31 & $82 \%$ \\
\hline D'accord, plutôt d'accord & 8 & $62 \%$ & 3 & $8 \%$ \\
\hline Ne sait pas & 2 & $15 \%$ & 4 & $10 \%$ \\
\hline
\end{tabular}

Tableau 12 : Modification des relations entre les services et les personnes pour traiter les informations obtenues via les NTIC Population 1 Population 2

\begin{tabular}{|l|c|c|c|c|}
\hline Pas d'accord, plutôt pas d'accord & 6 & $50 \%$ & 32 & $84 \%$ \\
\hline D'accord, plutôt d'accord & 6 & $50 \%$ & 2 & $5 \%$ \\
\hline Ne sait pas & 0 & & 4 & $11 \%$ \\
\hline
\end{tabular}

En cela, nos résultats rejoignent les conclusions de récentes publications qui soulignent que les entreprises développant une stratégie Internet ne peuvent faire l'économie de profondes modifications de leur organisation (Kalika, 2000).

Afin de prolonger les interprétations précédentes et de s'interroger sur les choix organisationnels liés aux NTIC, il semble intéressant de connaître le degré de diffusion des ces dernières parmi le personnel des organisations concernées.

Tableau 13 : Diffusion des NTIC dans l'entreprise : les acteurs ayant accès à Internet

\begin{tabular}{|r|c|c|c|c|}
\cline { 2 - 4 } \multicolumn{1}{c|}{} & \multicolumn{2}{c|}{ Population 1 } & \multicolumn{2}{c|}{ Population 2 } \\
\hline La majorité du personnel & 7 & $47 \%$ & 11 & $24 \%$ \\
\hline $\begin{array}{r}\text { Seulement certaines } \\
\text { personnes }\end{array}$ & 8 & $53 \%$ & 34 & $76 \%$ \\
\hline
\end{tabular}


A un premier niveau de lecture, remarquons que les entreprises déclarant valoriser les NTIC correspondent largement à celles ayant fait un effort sur l'accessibilité et la diffusion de ces dernières. Cette analyse paraît d'autant plus intéressante si on rappelle le nombre d'Intranets développés par les organisations des deux populations ici étudiées : 2 entreprises sur 3 de la population 1 disposent d'un Intranet pour moins de 1 entreprise sur 6 dans la population 2 . Or, les Intranets (Kalika, 2000 ; Laval, 2000) sont tenus sous certaines conditions comme l'un des outils de communication facilitant l'accès aux informations de l'entreprise, le travail en équipe

Une large diffusion des NTIC à l'intérieur de l'organisation semble faciliter l'accessibilité aux informations et l'interactivité dans certains relations interpersonnelles. Ces technologies peuvent donc être tenues pour l'un des facteurs jouant favorablement sur la perméabilisation des frontières hiérarchiques et fonctionnelles (Laval, 2000), sur l'élargissement des frontières organisationnelles (Benavent, 2000), voire sur la mise en ligne de l'organisation dans son ensemble (M. Kalika ${ }^{15}$, 2000).

A ce stade de l'étude, il est possible de développer nos interprétations précédentes à partir d'interrogations sur la construction de ressources par l'entreprise afin de promouvoir et favoriser l'utilisation des NTIC et sur les échanges spontanés d'information - obtenues via les NTIC - dans l'entreprise (respectivement, tableau 14 et tableau 15).

Tableau 14: Mise en place de ressources ou d'assistances lors de l'implantation et du développement des NTIC (possibilité de plusieurs réponses)

\begin{tabular}{|l|c|c|c|c|}
\cline { 2 - 5 } \multicolumn{1}{c|}{} & \multicolumn{2}{c|}{ Population 1 } & \multicolumn{2}{c|}{ Population 2 } \\
\hline Mis en place des plans de formation & 7 & $50 \%$ & 4 & $13 \%$ \\
\hline Financé des actions de formations ponctuelles & 4 & $29 \%$ & 8 & $26 \%$ \\
\hline Mis en place des assistances internes pour ceux qui en avaient besoin & 11 & $79 \%$ & 9 & $29 \%$ \\
\hline Laissé chacun trouver les aides dont il estimait avoir besoin & 4 & $29 \%$ & 16 & $52 \%$ \\
\hline Total répondants & 14 & & 31 & \\
\hline
\end{tabular}

Si plus d'une entreprise sur deux de la population 2 ont laissé les "problèmes se régler d'eux-mêmes" lors de l'implantation et du développement des NTIC ("laissé chacun trouver les aides dont il estimait avoir besoin"), près de quatre entreprises sur cinq de la population 1 ont "mis en place des assistances internes pour ceux qui en avaient besoin". Plus précisément, dans la première population, près de $90 \%$ des entreprises ont répondu favorablement à 2 des 3 items du tableau précédents. Cette proportion tombe à une entreprise sur 4 dans la population 2. Ici aussi, nous constatons que la valorisation des NTIC dans les entreprises de la population 1 est, pour une large part, concomitante, avec la construction (intentionnelle) de ressources multiples et variées (assistances internes, plans de formation). Sur ce point, nos résultats rejoignent ceux de recherches récentes (Laval, 2000 ; Osterman, 1995) soulignant qu'une participation précoce du personnel lors du processus de mise en place des NTIC se révélait utile pour l'organisation concernée et augmentait ses chances de succès quant à l'exploitation de ces technologies.

Il semble important de rapprocher ces derniers résultats avec ceux obtenus précédemment. On peut vérifier en effet que pour les entreprises de la population 1, le projet de se "connecter"

15 Précisément, M. Kalika remarque (p. 72) que si l'organisation en ligne peut être la source d'avantage concurrentiel, cette nouvelle forme organisation repose surtout sur la "mise en place d'Intra-extranet et l'on peut légitimement se demander si le système d'information ne va pas constituer l'essentiel de l'organisation de ces entreprises ». 
(via un site $W e b$, des applications de marketing ou de veille économique) à l'environnement "externe" est largement concomitant à la volonté de transformer l'organisation et de construire des ressources nécessaires aux acteurs pour s'approprier les NTIC.

Le tableau suivant, évoquant les échanges spontanés ou informels (d'acteur à acteur) d'information, corrobore aussi le raisonnement reliant la diffusion des NTIC au degré de communication (au-delà des frontières hiérarchiques et fonctionnelles) dans l'entreprise.

Tableau 15: Des personnes de l'entreprise échangent spontanément ou de façon informelle des informations obtenues via des NTIC

\begin{tabular}{|l|c|c|c|c|}
\cline { 2 - 5 } \multicolumn{1}{c|}{} & \multicolumn{3}{c|}{ Population 1 } & \multicolumn{2}{c|}{ Population 2 } \\
\hline Pas d'accord, plutôt pas d'accord & 1 & $8 \%$ & 25 & $64 \%$ \\
\hline D'accord, plutôt d'accord & 12 & $92 \%$ & 11 & $28 \%$ \\
\hline Ne sait pas & 0 & & 3 & $8 \%$ \\
\hline
\end{tabular}

Ces résultats prolongent les explications développées dans différentes recherches en management stratégique. En premier lieu, un grand nombre de travaux (Lesca, 1989; Marmuse, 1992 ; Martinet, 1991) rappellent que si les interactions et les échanges d'information dans l'entreprise semblent insuffisants, cela provient souvent de sa structure d'organisation. Il paraît en effet difficile d'entretenir une certaine interactivité selon le degré de parcellisation ou de spécialisation des activités, le cloisonnement des informations en fonction des niveaux hiérarchiques et des fonctions. Dans ce cas le degré de diffusion des NTIC n'est qu'un reflet des structures communicationnelles et professionnelles de l'entreprise. $C^{\prime} e s t$ en ce sens que l'on peut parler de capacités organisationnelles : ses structures sont héritées du passé et doivent être intégrées en tant qu'objet de la stratégie d'usage. A l'inverse, le fait d'impliquer et d'aider, dès le départ, un ensemble important d'acteurs (de la direction générale aux opérationnels) semble exercer une influence favorable sur la circulation des informations dans l'entreprise. L'accroissement des échanges spontanés d'information par les NTIC constatés serait le reflet de la participation d'un plus grand nombre d'acteurs au processus communicationnel.

Nos résultats prolongent ainsi ceux d'autres études menées sur des organisations ayant adopté des NTIC. Ces recherches soulignent ainsi que la communication médiatisée via les NTIC ne fonctionne que dans la mesure où chaque acteur perçoit un intérêt collectif et individuel, c'est-à-dire qu'il a « un rôle au sein de l'ensemble qui fait de lui un véritable relais permettant la circulation de la communication via le média à l'intérieur du réseau» (Comtet, 2000) ${ }^{16}$.

De façon générale, ces résultats semblent révéler la capacité organisationnelle et la volonté stratégique des entreprises de la population 1 de faire des NTIC une source de multiples avantages concurrentiels. Leur caractéristique principale est une mise en réseau au sens humain et technique avec différents acteurs économiques en même temps qu'elle considère comme normal l'usage généralisé en leur sein de cette technologie. On observe alors au sein de ces PME une synergie entre Internet et Intranet.

\section{CONCLUSION}

La capacité des PME à combiner, à partir de leur usage des NTIC, des réductions en terme de coûts et des innovations en terme de produits et services, peut être interprétée comme le

16 Caractères gras ajoutés par nous. 
résultat d'une interdépendance temporelle entre trois grands types de variables : la dynamique sectorielle du paradigme technologique, les stratégies d'usage mise en œuvre dans le cadre de stratégies génériques et les capacités organisationnelles de l'entreprise ${ }^{17}$. Ces capacités organisationnelles reposent à la fois sur une forme d'organisation du travail interne permissive d'une large diffusion des accès aux NTIC ainsi que sur des modalités de constitution des ressources et d'interactions avec des acteurs externes, au moyen de ces technologies. La technologie Internet vient alors faciliter au sein d'une structure d'échanges qui préexistait, à la fois la réduction des coûts de l'information ou des délais et la recherche d'information, voire l'acquisition de connaissances favorables à l'innovation. Elle devient pour les entreprises qui répondent à cet ensemble de condition structurelle une source d'avantages concurrentiels simultanément exprimable en termes de réduction des coûts, d'amélioration de la qualité et d'innovation de produits et services. Enfin, si l'hypothèse de rupture de paradigme technologique liée à l'avènement d'Internet peut être envisagée (Benghozi, Kavassalis et Solomon, 1996), il semble, du moins dans le cas des PME, qu'elle soit fortement liée à une nouvelle donne des activités de services.

\section{BIBLIOGRAPHIE}

S. Amabile, M. Gadille, R. Meissonier, «Information, organisation, décision : étude empirique sur les apports des NTIC dans des PME "Internautes" », Systèmes d'Information et Management, $\mathrm{n}^{\circ} 1$, volume 1, p. 41-60, mars 2000

C. Benavent, « Les NTIC, le marketing stratégique et le jeu concurrentiel », Revue Française de Gestion, nº129, p. 91-100, Juin-Juillet-Août 2000

P.J. Benghozi, P. Kavassalis, R.J. Solomon, «The internet: a paradigmatic rupture in cumulative telecom evolution », Industrial and corporte change, vol. 5, n4, 1996

P.J. Benghozi, « De l'organisation scientifique du travail à l'organisation scientifique du client. L'orientation-client, focalisation de nouvelles pratiques managériales », Réseaux, n91, p. 1329,1998

M. Bernasconi, «Les systèmes d'information interorganisationnels sont-ils toujours une source d'avantages concurrentiels durables ?», Systèmes d'Information et Management, $\mathrm{n}^{\circ} 1$, volume 1, p. 7-24, 1996

E. Brousseau, A. Rallet, Technologies de l'information, organisation et performances économiques, Rapport du groupe de travail présidé par E. Brousseau et A. Rallet, Commissariat Général au plan, mai 1999

A.D. Chandler, "Organizational Capabilities and the Economic History of the Industrial Entreprise », Journal of Economic Perspectives, Volume 6, n³, p. 79-100, summer 1992

I. Comtet, «Travail collaboratif médiatisé : le réseau social doit-il précéder le réseau technique ? », $5^{e}$ colloque de l'AIM, Montpellier, novembre 2000

D. Foray, J. Mairesse (eds.), Innovation et performances. Approches interdisciplinaires, Paris, Edition de l'Ecole des Hautes Etudes en Sciences Sociales, 1999

17 Cette notion a été développée au cœur du courant évolutionniste, sur la base des travaux anciens de Marshall, Shumpeter et Penrose, par Nelson et Winter (1982) ainsi que de Chandler, Teece, Dosi, et Lazonick. Elle repose sur l'idée de l'existence d'une hiérarchie de routines organisationnelles (définies dans le passé) qui déterminent largement ce que la firme est effectivement capable de faire (Nelson 1991). 
M. Gadille, A. d'Iribarne, « La diffusion d'Internet dans les PME : motifs d'adoption dans les réseaux et ressources mobilisées ", Réseaux, n¹04, volume 18, 2000

J.L. Gaffard, Economie industrielle et de l'innovation, Dalloz, 1990

G. Hamel, C.K. Prahalad, « Strategic Intent », Harvard Business Review, May-June, 1989

M. Kalika, «Le management est mort, vive le e-management!», Revue Française de Gestion, n²129, p. 68-74, juin-juillet-août 2000

F. Laval, "Gestion des ressources humaines et NTIC : enjeux et perspectives », Revue Française de Gestion, ${ }^{\circ} 129$, p. 80-90, juin-juillet-août 2000

J.-L. Le Moigne, «Vers un système d'information organisationnel ? », Revue Française de Gestion, novembre-décembre 1986

H. Lesca, Information et adaptation de l'entreprise, Masson, Paris, 1989

J.G. March, "Exploration and Exploitation in Organizational Learning », Organization Science, vol. 2, n 1, p. 71-87, february 1991

C. Marmuse, Politique Générale. Langages, Intelligence, Modèles et Choix Stratégiques, Economica, Paris, 1992

A.-C. Martinet, «Management en temps réel et continuité stratégique sont-ils compatibles ? », Revue Française de Gestion, novembre-décembre 1991

R.R. Nelson, «Why Firms Differ, and How Does it Matter? », Stratégic Management Journal, vol. 12, p. 61-74, winter 1991

R.R. Nelson, S.G. Winter, An Evolutionary Theory of Economic Change, Cambridge : Harvard University Press, 1982

P. Osterman, «Impact des technologies de l'information sur les postes de travail et les spécialisations du personnel », in M.S. Scott Morton (ed), L'entreprise compétitive au futur, technologies de l'information et transformation de l'organisation, Editions d'Organisation, Paris, 1995

Paturel R. , "Les manœuvres stratégiques génériques des entreprises », In Economies et Sociétés, Sciences de Gestion, $\mathrm{n}^{\circ}$ 7-8/1997, p.93-118.

M.J. Piore, F.S. Sabel, The second Industrial Divide, Basic Books, Inc., Publishers, New York, 1984

M. Porter, Competitive advantage : creating and sustaining superior performance, The Free Press, Macmillan, Inc. New-York, 1985

M. Porter, V.E. Millar, «How Information gives you competitive advantage », Harvard Business Review, July-August, 1985

N. Rosenberg, "Learning by using », in Inside the Black Box by N. Rosenberg, Cambridge University Press, cambridge, 1982

D. Teece, G. Pisano, «The dynamic capabilities of the firms : an introduction », in Technology, Organization, and Competitiveness » edited by G. Dosi, D.J. Teece, and J. Chitry, p. 193-212, 1998 